\section{Trois petites (nano) \\ controverses et leurs morales}

\section{Raphaël Lévy}

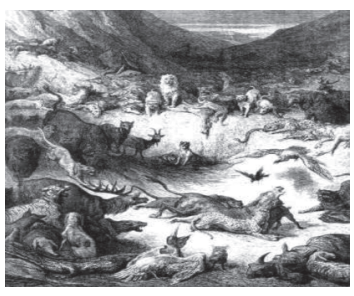

Institute of Integrative Biology, university of Liverpool, Crown street, L69 7ZB, Liverpool, Royaume Uni rapha@liverpool.ac.uk

La quasi-absence de discussions contradictoires dans la littérature scientifique contemporaine de nombreux domaines pourrait nous le faire oublier, mais les controverses sont un aspect essentiel du progrès de la connaissance permettant d'identifier les points faibles de travaux et ainsi, de les renforcer ou les invalider de manière concluante [6]. Elles sont consubstantielles à l'exercice des sciences [7]. Leur analyse peut aussi être un moyen de «cartographier symétriquement » les acteurs pour mieux comprendre les individus et les processus sociaux qui entrent en jeu [8]. Dans ce forum, je décris trois controverses récentes dans mon domaine de recherche, les nanoparticules d'or appliquées à la biologie et à la médecine. II ne s'agit pas d'une cartographie symétrique : je n'en suis pas un observateur neutre, mais un scientifique actif à divers degrés dans chacune d'entre elles. J'essaie néanmoins d'en tirer quelques enseignements et suggestions pratiques pour améliorer notre pratique de la science.

\section{Nanoparticules Zèbres}

En 2004, le groupe de Francesco Stellacci, au Massachusetts Institute of Technology (MIT) aux États-Unis, publie dans la prestigieuse revue Nature Materials un article décrivant des nanoparticules d'or recouvertes par un mélange de deux molécules qui s'auto-organisent pour former des rayures observées par microscopie à force atomique [9]. Cet article et les nombreux autres qui vont suivre dans la même revue, et d'autres toutes aussi prestigieuses comme le Journal of the American Chemical Society (JACS) [10], Science [11] et Proceedings of the National Academy of Sciences (PNAS) [12], suggèrent que, grâce à leurs zébrures, ces nanoparticules ont des propriétés uniques en terme de mouillage, d'auto-organisation, d'interactions avec les protéines et de pénétration dans les cellules, avec des possibilités nombreuses d'application pour la détection biomoléculaire, voire l'administration de médicaments. Ces articles contribuent certainement à l'avancée de 
la carrière des auteurs, mais les rayures sont un artefact expérimental bien connu des utilisateurs de microscopie à force atomique ${ }^{1}$. Dès lors, comment se fait-il que plus de 20 articles «zèbres » aient été publiés entre 2004 et 2012 ? II est évident que des spécialistes (ou même des amateurs éclairés) avaient identifié le problème dès 2004. Pourtant, les articles et revues de l'époque n'en montrent aucune trace. On sait aujourd'hui que Predrag Djuranovic a été le premier à entreprendre un travail scientifique visant à tester, et éventuellement remettre en question, l'existence des zébrures. En 2005, ce rigoureux et courageux scientifique était un étudiant dans le laboratoire de Francesco Stellacci au MIT. Ses résultats expérimentaux, ainsi qu'un modèle numérique montrant comment les zébrures apparaissent lorsque le circuit de contrôle du microscope est mal réglé, étaient sans appel, mais le MIT s'assura que ces résultats demeureraient secrets [13]. En 2007, je soumis un commentaire («technical comment ») répondant à l'article de Science. Cette première tentative, limitée aux conclusions de l'article de Science, fut infructueuse: Science demanda à Francesco Stellacci une réponse mais décida de ne pas publier l'échange [14]. En 2008, un nouvel article du groupe du MIT, à nouveau dans Nature Materials, rapporte que, grâce à leurs zébrures, ces nanoparticules peuvent traverser la membrane cellulaire pour accéder directement au cytoplasme [15]. Un commentaire accompagnant l'article s'intitule Particles slip cells security [16]. Après discussions avec plusieurs de mes étudiants, nous décidons de proposer une réponse plus exhaustive. Quelques mois plus tard, l'article «Stripy nanoparticles revisited » est prêt. II inclut une nouvelle analyse des images «zèbres » concluant que les oscillations sont un artefact, et une discussion critique des propriétés physico-chimiques et biologiques accompagnée de nouveaux résultats expérimentaux remettant en cause la capacité de ces particules à accéder directement à l'intérieur de la cellule. L'article est soumis d'abord à Nature Materials (où il a été refusé) puis à NanoLetters (également refusé) puis à Small... où il sera publié à la suite d'un processus éditorial qui durera trois ans [1719]. La publication de l'article, en novembre 2012, ne met pas fin à la controverse. Elle s'étend (un peu) dans la littérature scientifique et elle prend aussi d'autres formes (en particulier sur mon blog et d'autres [2023]). Des problèmes de réutilisation d'images par Francesco Stellacci émergent et débouchent sur deux corrections (concernant la référence [15] et la référence [12]). Après bien des efforts, Philip Moriarty et Julian Stirling (School of Physics and Astronomy, university of Nottingham, Nottingham, Royaume Uni) obtiennent l'accès aux données originales de l'article de 2004. Ils démontrent alors (entre autres choses) que les zébrures sont présentes dans toute l'image, y compris entre les particules d'or [24], une conclusion toujours rejetée par Francesco Stellacci [25].

\section{Nanoparticules homéopathiques}

Le laboratoire de Molly Stevens à I'Imperial College de Londres est l'un des plus prestigieux du monde dans le domaine des biomatériaux. En

Le microscope à force atomique offre une image topographique de la surface de l'objet à l'échelle nanométrique.
2012, deux articles du groupe décrivent les propriétés particulièrement intéressantes des nanoparticules d'or pour les applications de diagnostic. Le premier, publié dans Nature Materials, rapporte un phénomène tout à fait extraordinaire dans lequel le signal mesuré augmente quand la concentration de molécules à détecter diminue («inverse sensitivity») [26]. Encore plus incroyable: ce phénomène continue jusqu'à une concentration qui correspond à moins d'une molécule d'enzyme, en moyenne, dans le volume étudié. Le deuxième article paru dans Nature Nanotechnology va encore plus loin: plus besoin d'instrument, la détection de concentrations du même ordre est alors obtenue grâce à un changement de couleur visible à I'œil nu [27]. Ces deux articles font l'objet de critiques sévères et détaillées sur le site PubPeer ${ }^{2}$ [28, $29]$; les objections sont simples et profondes mais les auteurs n'ont pas souhaité répondre (le lecteur intéressé pourra lire les critiques et les articles en ques$\left.\operatorname{tion}^{3}\right)$. On notera que le nombre d'Avogadro comporte beaucoup de zéros (630000000000000000000000) et que la détection d'un changement de propriétés macroscopiques, provoqué par une molécule unique, est donc une prouesse qui demande des preuves extrêmement solides. L'une des critiques sur le site PubPeer indique que l'éditeur de Nature Nanotechnology a été contacté en janvier 2013, mais quatre ans plus tard rien n'indique le moindre doute concernant cet article ni sur le site de Nature Nanotechnology, ni dans la littérature scientifique traditionnelle, ni dans les journaux de la presse grand public comme Le Monde et le Daily Mail qui, pourtant, avaient couvert cette publication lors de sa parution $[30,31]$.

\section{L'ADN sphérique}

Le laboratoire de Chad Mirkin à l'université Northwestern aux États-Unis, est l'un des plus prestigieux du monde dans le domaine des nanosciences appliquées à la biologie et à la médecine. Un axe majeur de sa recherche est I' «ADN sphérique » ou «spherical nucleic acids », un terme introduit par Mirkin pour décrire les nanoparticules d'or fonctionnalisées avec des brins d'ADN (ou d'ARN). Ces ADN sphériques présenteraient de nombreuses propriétés, différentes de celles des acides nucléiques linéaires [32]. En particulier, ils seraient capables d'accéder au cytosol des cellules vivantes et de détecter et réguler, dans ces cellules,

\footnotetext{
${ }^{2}$ PubPeer est un site qui permet d'examiner et de discuter les résultats publiés.

${ }^{3}$ Une critique détaillée a été publiée par Boris Barbour, institut de biologie de l'école normale supérieure, CNRS UMR 8197, Inserm U1024, PSL research university, en mai 2017 sur le site de Preprint Arxiv : https://arxiv.org/abs/1705.09509.
} 
la présence et la quantité d'ARN messagers. On peut se demander pourquoi cette solution n'est pas apparue au cours de l'évolution: pour accéder à la machinerie cellulaire, il aurait ainsi suffi aux virus et aux bactéries de s'emballer dans leur propre ADN pour cibler la machinerie de la cellule qu'ils infectent. Les premiers articles, parus dans Science [33], le Journal of the American Chemical Society [34], Nanoletters [35], ACS Nano [36], qui proposent cette étonnante théorie, ne mentionnent pas du tout le mécanisme d'entrée des particules dans la cellule. Les suivants (par exemple [37]) proposent un processus d'endocytose, mais ils n'expliquent pas le mécanisme par lequel les particules s'échapperaient des endosomes. Après plusieurs dizaines d'articles sur le sujet, la proportion de particules qui atteindrait la machinerie cellulaire n'est toujours pas connue (pourtant, les nanoparticules d'or sont utilisées depuis les années 1950, comme agent de contraste pour étudier le trafic cellulaire; une telle étude ne serait donc pas très difficile). Un article du groupe de Chad Mirkin suggère néanmoins que les ADN sont dégradés dans les endosomes et qu'au maximum, une très faible proportion s'en échappe ( $A$ small, unquantifiable portion escape... », [38]). Malgré cette faible proportion, les particules sont désormais commercialisées sous le nom de SmartFlares (Merck Millipore) pour détecter l'ARN dans les cellules. Nous avons étudié l'entrée de ces particules dans la cellule et leur capacité à détecter l'ARN. Échaudés par les nanoparticules zèbres, nous avons adopté une stratégie différente. Le projet a été ouvert et nous avons partagé nos résultats et analyses quasiment en temps réel sur notre blog. En contradiction avec les descriptions faites par le fabricant et les articles de Mirkin, nous avons observé que les Smartflares étaient dégradés dans les endosomes et ne détectaient pas I'ARN. Comme dans le cas de Predrag Djuranovic et des nanoparticules zèbres, nous n'étions pas les premiers à avoir des doutes: Luke Armstrong, qui était chargé de développer cette technologie chez Merck Millipore en Californie (avant de quitter l'entreprise) était parvenu à la même conclusion [39]. Par souci de transparence et de rapidité, nous avons publié notre article sur la plate-forme (peu prestigieuse) ScienceOpen où l'expertise par les pairs est réalisée après publication [40]. Nous avons invité Chad Mirkin à le commenter, sans réponse. Un autre article récent de la même équipe, dans PNAS, décrit une nouvelle version de ces ADN sphériques pour la détection d'ARN [41]. Notre analyse des données brutes (obtenues de l'auteur après quelques efforts) montre que le signal détecté provient des endosomes. Notre lettre soumise à PNAS précisant cette controverse a été rejetée par le comité éditorial car « elle ne contribuait pas de manière significative à la discussion de l'article $»^{4}$.

\section{Morale}

L'accès aux données brutes est essentiel et garanti par des règles claires des universités, journaux scientifiques et agences de financement. Il est donc en général possible d'y accéder avec quelques efforts. Il est bien entendu préférable de publier ces données en même

${ }^{4}$ Cette lettre est disponible sur le serveur de preprint BioRxiv [42]. temps que les articles. Ceci est d'ores et déjà la norme pour certains types de résultats et devrait se généraliser. Les chercheurs devraient également adopter le manifeste pour une recherche reproductible (Manifesto for reproducible research [43]). Les outils sont en place afin d'améliorer la pratique de la science.

Les évaluations de la science et des scientifiques doivent impérativement être fondées sur une analyse critique et des résultats probants, non sur le prestige des institutions et des revues scientifiques dans lesquelles sont publiés les articles. Ceci nécessite un changement d'attitude et un engagement clair des chercheurs en position de pouvoir (c'est-à-dire ceux qui participent aux processus de recrutements ou de promotions). Dire qu'un article est un bon article parce qu'il a été publié dans une revue prestigieuse est une faute logique et morale qui doit être contestée.

Les institutions et revues scientifiques ne sont pas mues par la recherche de la vérité. Les décisions prises par le MIT (ne pas rendre public les travaux de Predrag Djuranovic), par Nature Materials (ne pas publier l'article «Stripy Nanoparticles Revisited »), par Science (ne pas publier les échanges avec Francesco Stellacci [14]), et par PNAS (ne pas publier l'article [42]), ont directement impacté les avancées de la connaissance. Ces institutions affichent des principes recommandables, mais, dans la pratique, leur application vise surtout à protéger leur réputation et donc leurs financements [44]. Cet objectif ne s'aligne que partiellement avec le progrès scientifique qui nécessite, lui, une discussion rapide et ouverte des résultats et des conclusions. Le web, inventé pour le partage de la science, permet cette discussion. Le chercheur aura donc à se familiariser avec ces puissants outils : 1) PubPeer pour commenter les articles; 2) Les preprints qui permettent de publier rapidement, de minimiser l'influence des éditeurs scientifiques, et de dissocier la publication (c'est-àdire le partage de l'information) de l'évaluation par les pairs; 3) Les réseaux sociaux, par exemple Twitter ou les blogs, qui constituent une conférence scientifique permanente pour discuter des expériences, des résultats, des méthodes, des analyses et des nouvelles publications. $\diamond$

Three small (nano) controversies and their morals

\section{REMERCIEMENTS}

Je remercie Marianne Noel (IFRIS) pour sa lecture critique de cet article et Marianne Lévy pour ses commentaires sur la grammaire et le style, bien nécessaires après 14 années passées dans un pays anglophone...

\section{LIENS D'INTÉRÊT}

L'auteur déclare n'avoir aucun lien d'intérêt concernant les données publiées dans cet article. 


\section{RÉFÉRENCES}

1. La Fontaine J (de). Les animaux malades de la peste. Fables, 1678.

2. loannidis JPA, Boyack K, Klavans R, et al. How to make more published research true. PLoS Med $2014 ; 11$ : e1001747.

3. Baker M, Dolgin $\varepsilon$. Cancer reproducibility project releases first results. Nature 2017 ; 541 : 269-270.

4. Laframboise D. How many scientific papers just aren't true? The Spectator 2016.

5. Krauss LM. Donald Trump's war on science. New Yorker 2016.

6. Dascal M. The study of controversies and the theory and history of science. Sci Context 1998 ; $11: 147$.

7. Latour B. Pasteur et Pouchet : hétérogenèse de l'histoire des sciences (sous la direction de Michel Serres). Éléments d'histoire des sciences 1989 : 423-45.

8. Pestre D. L'analyse de controverses dans l'étude des sciences depuis trente ans. Mil neuf cent. Rev d'histoire Intellect $2007 ; 25: 29-43$.

9. Jackson AM, Myerson JW, Stellacci F. Spontaneous assembly of subnanometre-ordered domains in the ligand shell of monolayer-protected nanoparticles. Nat Mater $2004 ; 3: 330-6$.

10. Jackson AM, Ying Hu Y, Silva PJ, Stellacci F. From homoligand- to mixed-ligand- monolayerprotected metal nanoparticles: a scanning tunneling microscopy investigation. J Am Chem Soc $2006 ; 128: 11135-47$.

11. DeVries GA, Brunnbauer M, Hu Y, et al. Divalent metal nanoparticles. Science 2007 ; 315

12. Centrone A, Penzo $\varepsilon$, Sharma M, et al. The role of nanostructure in the wetting behavior of mixedmonolayer-protected metal nanoparticles. Proc Natl Acad Sci USA 2008 ; 105 : 9886-91.

13. Djuranovic P. Seven years of imaging artifacts: what gives? Rapha-Z-Lab 2012.

14. Levy R. Divalent metal nanoparticles. PubPeer. https://pubpeer.com/publications/4DA88768C5B2 79ع24ع469CC0080A47

15. Verma A, Uzun 0 , Hu Y, et al. Surface-structure-regulated cell-membrane penetration by monolayer-protected nanoparticles. Nat Mater $2008 ; 7: 588-95$

16. Xia T, Rome L, Nel A. Nanobiology: particles slip cell security. Nat Mater $2008 ; 7: 519-20$.

17. Cesbron Y, Shaw CP, Birchall JP, et al. Stripy nanoparticles revisited. Small $2012 ; 8: 3714-9$.

18. Yu M, Stellacci F. Response to "Stripy nanoparticles revisited". Small $2012 ; 8: 3720-6$.

19. Levy R. Stripy timeline. 2012. Rapha-z-lab https://raphazlab.wordpress.com/2012/12/20/stripytimeline/

20. Dove A. Do these stripes make my nanoparticles look weird? 2012. http://alandove.com/ static/2012/12/do-these-stripes-make-my-nanoparticles-look-weird/

21. Fernig DG. Ferniglab Blog. https://ferniglab.wordpress.com/?s=stripy

22. Neuroskeptic. Science is interpretation. Discov Mag Blogs 2014. http://blogs.discovermagazine. com/neuroskeptic/2014/01/04/reanalysis-science/

23. Natelson D. A nano-controversy. Nanoscale views 2012. http://nanoscale.blogspot. co.uk/2012/12/a-nano-controversy.html

24. Stirling J, Lekkas I, Sweetman A, et al. Critical assessment of the evidence for striped nanoparticles. PLoS One 2014 ; 9 : el08482.

25. Ong QK, Stellacci F, Jeschke G, et al. Response to "Critical Assessment of the Evidence for Striped Nanoparticles." PLoS One 2015 ; 10 : e0135594.

26. Rodríguez-Lorenzo L, la Rica R de, Álvarez-Puebla RA, et al. Plasmonic nanosensors with inverse sensitivity by means of enzyme-guided crystal growth. Nat Mater $2012 ; 11: 604-7$

27. la Rica R de, Stevens MM. Plasmonic ELISA for the ultrasensitive detection of disease biomarkers with the naked eye. Nat Nanotechnol $2012 ; 7: 821-4$
28. PubPeer "Plasmonic nanosensors with inverse sensitivity by means of enzyme-guided crystal growth". https://pubpeer.com/ publications/3E8208F0654769A44C22D4E78DA2B8

29. PubPeer "Plasmonic ELISA for the ultrasensitive detection of disease biomarkers with the naked eye". https://pubpeer.com/publications/54AECF 24ع96162ع3A563AED08BE0B3

30. Des nanoparticules d'or pour dépister VIH ou cancer à l'œil nu. Le Monde 2012.

31. Bates C. Colour-coded blood test that turns blue if you have HIV is 10 times more sensitive than current methods. The Daily Mail 2012.

32. Cutler Jl, Auyeung $\varepsilon$, Mirkin CA. Spherical nucleic acids. J Am Chem Soc 2012 ; 134 : 1376-91.

33. Rosi NL, Giljohann DA, Thaxton CS, et al. Oligonucleotide-modified gold nanoparticles for intracellular gene regulation. Science 2006 ; 312 : 1027-30.

34. Seferos DS, Giljohann DA, Hill HD, et al. Nano-flares: probes for transfection and mRNA detection in living cells. J Am Chem Soc 2007 ; 129 : 15477-9.

35. Zheng D, Seferos DS, Giljohann DA, et al. Aptamer nano-flares for molecular detection in living cells. Nano Lett 2009 ; 9 : 3258-61.

36. Prigodich AE, Seferos DS, Massich MD, et al. Nano-flares for mRNA Regulation and Detection. ACS Nano 2009 ; 3 : 2147-52.

37. Choi CHJ, Hao L, Narayan SP, et al. Mechanism for the endocytosis of spherical nucleic acid nanoparticle conjugates. Proc Natl Acad Sci USA $2013 ; 110: 7625-30$

38. Wu XA, Choi CHJ, Zhang C, et al. Intracellular fate of spherical nucleic acid nanoparticle conjugates. J Am Chem Soc 2014 ; 136 : 7726-33.

39. Schneider L. Do nanoparticles deliver? Merck's Smart Flares and other controversies, 2015. https://forbetterscience.com/2015/11/20/ do-nanoparticles-deliver-mercks-smart-flares-and-other-controversies/

40. Mason D, Carolan G, Held M, et al. The spherical nucleic acids mRNA detection paradox. ScienceOpen Res 2016 ; DOI: 10.14293/S21991006.1.SOR-CHEM.AZ1MJU.vl.

41. Briley WE, Bondy MH, Randeria PS, et al. Quantification and real-time tracking of RNA in live cells using Sticky-flares. Proc Natl Acad Sci USA $2015 ; 112: 9591-5$.

42. Mason D, Levy R. Sticky-flares: real-time tracking of mRNAs. or of endosomes? bioRxiv 2015. http://biorxiv.org/content/ early/2015/10/19/029447

43. Munafò MR, Nosek BA, Bishop DVM, et al. A manifesto for reproducible science. Nat Hum Behav $2017 ; 1: 21$.

44. Glanz J, Armendariz A. Years of ethics charges, but star cancer researcher gets a pass. New York Times 2017.

TIRÉS À PART

R. Lévy

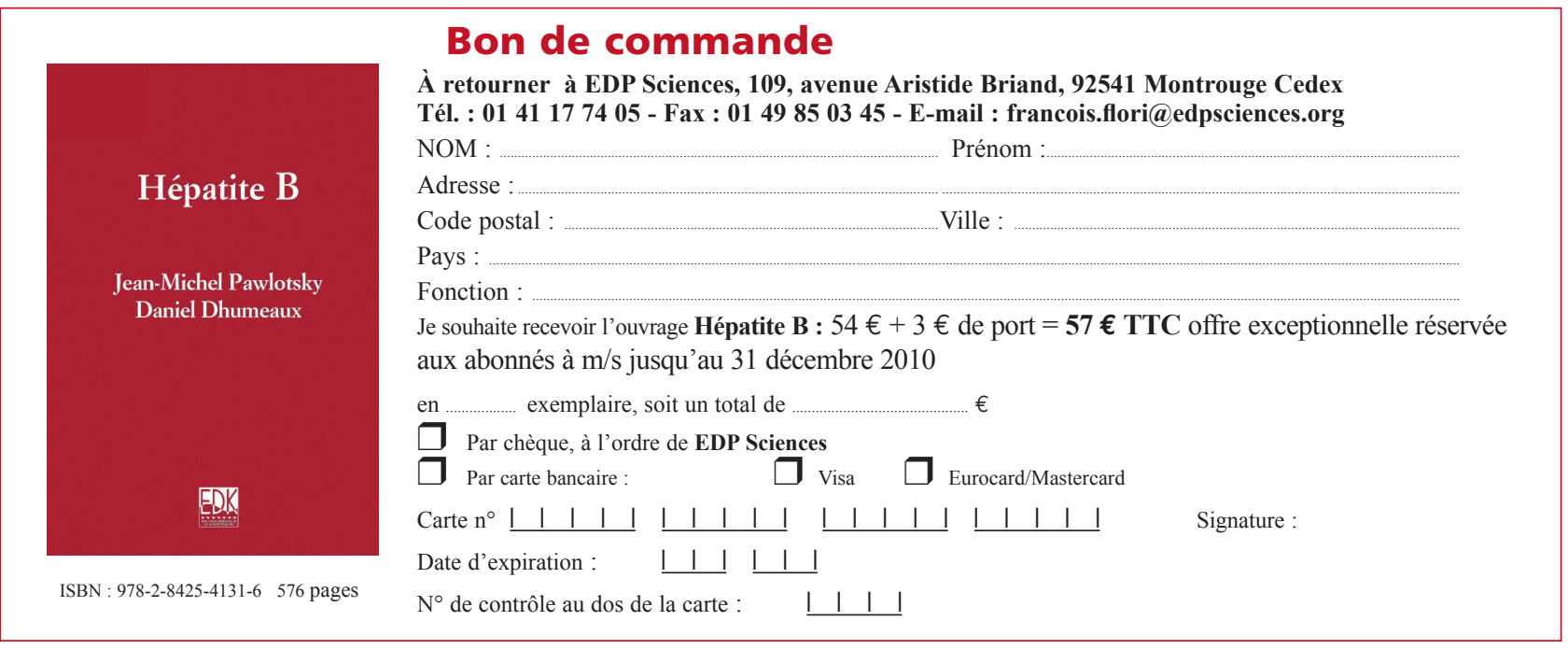

